\title{
Understanding protein multifunctionality: from short linear motifs to cellular functions
}

Andreas Zanzoni ${ }^{1}$, Diogo M. Ribeiro ${ }^{1}$, Christine Brun ${ }^{1,2, \#}$
${ }^{1}$ Aix-Marseille Univ, INSERM, TAGC, UMR_S1090, Marseille, France;
${ }^{2}$ CNRS, Marseille, France.

\# Address correspondence to: Christine Brun (christine-g.brun@inserm.fr).

Running title: SLiMs and protein multifunctionality 


\section{Abstract}

Moonlighting proteins perform multiple unrelated functions without any change in polypeptide sequence. They can coordinate cellular activities, serving as switches between pathways and helping to respond to changes in the cellular environment. Therefore, regulation of the multiple protein activities, in space and time, is likely to be important for the homeostasis of biological systems. Some moonlighting proteins may perform their multiple functions simultaneously while others alternate between functions due to certain triggers. The switch of the moonlighting protein's functions can be regulated by several distinct factors, including the binding of other molecules such as proteins. We here review the approaches used to identify moonlighting proteins, existing repositories. We particularly emphasise the role played by short linear motifs and PTMs as regulatory switches of moonlighting functions.

Keywords: moonlighting proteins; multifunctional proteins; cell signalling regulation; short linear motifs; molecular switch; protein interaction networks; bioinformatics. 


\section{Introduction}

In 1941, the seminal work of Beadle and Tatum led to the "one gene-one function" concept. Accordingly, each gene would encode a single protein, and that each protein would have a single function [1]. However, we now know that a single gene can encode different proteins (e.g., through alternative splicing), thus increasing the number of functions it can provide. Likewise, the discovery of moonlighting proteins brought to light the fact that multiple unrelated functions can be performed by a single protein, with no change in polypeptide sequence [2].

The term 'moonlighting proteins' was coined by Constance Jeffery in 1999 [3], by analogy to moonlighting in the sense of holding a second job in addition to a regular one. Under a strict definition, moonlighting proteins perform multiple functions "without partitioning these functions into different protein domains" [4]. However, less stringent definitions of moonlighting proteins have been recently used, adopting terms such as 'multitask proteins' and 'extreme multifunctional proteins', focusing on the fact that the proteins are indeed involved in diverse unrelated functions, regardless of their evolutionary history or domain organisation [5-7]. In this view, moonlighting functions may be performed by any region of the protein surface, often involving regions other than the one responsible for the canonical function [8].

It has been proposed that moonlighting proteins can coordinate several cellular activities, serving as switches between pathways and helping to respond to changes in the cellular environment $[3,9]$. Therefore, regulation of the multiple protein activities, in space and time, is likely to be important for the homeostasis of biological systems. Some moonlighting proteins may perform their multiple functions simultaneously, and each of these functions may be independently regulated, while other moonlighting proteins alternate between functions due to certain triggers. The switch of the moonlighting protein's functions can be triggered or regulated by several distinct factors, including the binding of another molecule 
such as another protein, sometimes in combination to each other [3,9]. These include the oligomeric state or post-translational modifications (PTMs) of the protein, the cell type or tissue where it is expressed, the cellular location where it is present, or even the cellular concentration of other molecules [10].

Although the cellular regulatory role of such proteins is undoubtedly important for cell homeostasis, the prevalence of moonlighting proteins in our proteome is still unknown. Herein, we briefly review some approaches used to identify moonlighting proteins and their functions, as well as the existing repositories of moonlighting and extreme multifunctional proteins. We particularly emphasize on the role played by sequence motifs and PTMs as regulatory switches of moonlighting functions. Indeed, we illustrate that, in numerous cases, these are responsible for the drastic functional changes characterizing moonlighting proteins.

\section{Detection of moonlighting and multifunctional proteins}

\section{Experimental approaches to detect moonlighting proteins}

The discovery of moonlighting functions has been largely serendipitous, by finding that two proteins serving distinct functions are in fact the very same protein [8]. For instance, moonlighting functions can be revealed experimentally when mutation or deletion studies result in unexpected phenotypes. Indeed, unequivocal identification of moonlighting proteins requires several studies, in order to show that some mutations affect both functions of the protein, while others affect only one [11]. However, such analysis would only work if the two functions use different parts of the protein and are not context-dependent. Providing evidence that several functions are indeed completely unrelated and not due to pleiotropic effects can be difficult.

An illustrative example is the recent study of Espinosa-Cantú and colleagues, in which they experimentally addressed the prevalence of enzymes with moonlighting functions in 
Saccharomyces cerevisiae [12]. They evaluated if the enzyme gene deletion phenotypes are caused solely by the loss of catalytic activity, or to a yet unknown moonlighting function independent of the catalytic activity [12]. This study showed that 4 out of 11 tested enzymes may have moonlighting functions, thus suggesting that moonlighting proteins may be highly prevalent. As these experiments were performed under constant conditions, they are unable to find cases of moonlighting proteins that only display alternative functions when present in different contexts. Consequently, the number of identified moonlighting proteins is probably an underestimate.

\section{Computational approaches to detect candidate moonlighting proteins}

Although the number of experimentally determined moonlighting proteins is increasing, their full prevalence is still unknown. For this reason, over the last years, a few large-scale computational methods to predict moonlighting proteins have been developed. They predicted hundreds to thousands of such proteins by using indicators and approaches such as sequence similarity [13], text mining [14], and machine learning classifiers exploiting features such as gene expression and structural disorder [15]. However, these methods may have shortcomings. For example, moonlighting may not be readily identified through sequence analysis, since multifunctionality may blur the results of sequence similarity searches [5]. Moreover, gene expression analyses are unable to find moonlighting proteins whose function differs upon cellular export and subcellular localisation changes, such as the human RHAMM protein (also known as HMMR) [16].

In 2015, we developed an innovative approach that, for the first time, combined proteinprotein interaction (PPI) network analysis and Gene Ontology (GO) functional annotations, to predict 'extreme multifunctional' (EMF) proteins on a whole proteome scale [5]. Since multifunctional proteins interact with different sets of proteins to perform their different cellular functions, the usage of the PPI network topology for their identification is pertinent [17]. In addition, we proposed novel metrics to identify functions that are highly dissimilar to each 
other, a hallmark of protein moonlighting [18]. The human EMF proteins predicted were defined as proteins "whose multiple functions are very dissimilar to one another", thus related to moonlighting proteins, but not constricted by the definition of moonlighting proteins which is also linked to the evolutionary history of the protein [6].

\section{Regulation of moonlighting and extreme multifunctional protein}

\section{functions}

Besides the difficult task of identifying moonlighting proteins, discovering how their unrelated functions are regulated and how they switch function is also challenging. Over the years, several triggers and regulatory mechanisms have been proposed such as changes in subcellular localisation, effector molecule concentration, structural conformation or oligomeric state $[3,9]$. Interestingly, several pieces of evidence, including our EMF analysis, suggested that another important clue may reside within short linear motifs (SLiMs) in the protein sequence.

\section{Regulatory potential of short linear motifs}

SLiMs are relatively conserved sequence stretches of around 3 to 10 residues, often occurring within an intrinsically disordered region of a protein [19]. They embody functional sites involved in protein modification and cleavage as well as subcellular targeting and protein interactions [20]. Given the limited number of residues contacting their binding partner, SLiM-mediated interactions are rather weak, with affinity often in the low micromolar range, meaning that they are transient and reversible [19]. Consequently, SLiM interaction specificity and affinity can be easily modulated by post-translational modifications (PTMs), which can have a significant effect on the interaction of a motif with its interaction partner [21, 
22]. In addition, motif specificity can also be determined by the local abundance of the interactors and their subcellular localisation [23].

Importantly, distinct SLiMs may co-occur within a given disordered region and even overlap [22]. However, these interactions are often mutually exclusive, allowing the protein to carry out different functional roles depending on the available partners, which may be important for moonlighting proteins. This could provide a key mechanism to switch between different cellular processes in a context-dependent manner through a change in protein interaction partners [24, 25], often modulated by PTMs (e.g., [26]).

\section{Short linear motifs as a molecular signature of extreme multifunctionality}

In our attempt to characterize extreme multifunctional proteins, we discovered several features that set them apart from other proteins and that shed a new light on the possible molecular mechanisms leading to their functional change. A typical EMF protein is more likely to have a high number of protein partners within a protein interactome, and to be expressed ubiquitously, suggesting that they can perform distinct functions in different tissues [5]. Importantly, EMF proteins were found to be significantly enriched in SLiMs. Moreover, we detected among EMF proteins a significant over-representation of proteins whose functions are regulated by motif-based molecular switches according to the Switch.ELM resource [27]. This finding suggests that such SLiMs could provide the ability for extreme multifunctional and moonlighting proteins to switch between functions.

\section{Involvement of short linear motifs in functional changes}

Increasing evidence in the literature indicates that changes in protein subcellular localisation and function are mediated by short linear motifs, particularly PTM sites (Table 1). For instance, the glyceraldehyde-3-phosphate dehydrogenase (GAPDH), which acts as a glycolytic enzyme in the cytosol, performs alternative functions when present in other subcellular locations such as the nucleus, when cells are exposed to a stressor [28]. Indeed, 
upon oxidative stress, GAPDH is S-nitrosylated and binds the E3-ubiquitin-ligase SIAH1, which bears a nuclear localisation signal (NLS). Subsequently, this complex translocates to the nucleus [29] where GAPDH is further acetylated by the acetyltransferase p300/CREB binding protein (CBP) [30]. Nuclear GAPDH stimulates the acetylation and catalytic activity of p300/CBP, thus triggering cell death [30]. In this example, a cascade of consecutive PTMs and protein interactions ultimately leads to the functional change of GAPDH. However, PTMs do not only alter the ability to bind proteins, but also chemical compounds and nucleic acids. An illustrative example is the human aconitase (ACO1), an enzyme of the tricarboxylic acid (TCA) cycle. When cellular iron concentration is low, the aconitase loses its interaction with a Fe-S cluster, changes its conformation and can bind iron responsive elements (IREs) in the mRNAs of iron metabolism genes, thus modulating their translation [31]. However, this switch of function can also be regulated by serine phosphorylation events [32], which favour the RNA-binding form [33] and destabilise the Fe-S cluster [33, 34].

In the case of the human phosphoglycerate kinase 1 (PGK1), a glycolytic enzyme that moonlights during tumour angiogenesis [35], early studies showed that it can bind the mRNA of the urokinase plasminogen activator surface receptor (PLAUR) and regulate its expression. This is mediated by the phosphorylation of a PGK1 tyrosine residue (Y76), that reduces the binding affinity of PGK1 to PLAUR mRNA, resulting in its stabilization and expression induction [36].

Besides metabolic enzymes, other classes of moonlighting proteins have been found to be regulated by PTMs. Indeed, several proteins involved in clathrin-mediated endocytosis $(\mathrm{CME})$ are associated with the successful completion of mitosis at distinct mitotic stages [37, 38]. Notably, these secondary roles are not dependent on their endocytic functions [38]. For instance, the large GTPase dynamin (DNM2), which is implicated in endocytic membrane remodelling events [39], is recruited to the spindle midzone [40] and participates in cytokinesis in a phosphorylation-dependent manner [38, 41]. 
Finally, experimental evidence shows that several proteins reside both in the nucleus and in the mitochondrion [42]. One of them is the TERF1-interacting nuclear factor 2 (TINF2, also known as the TIN2 protein), which interacts with TERF1, TERF2 and ACD (also known as TPP1) within the shelterin complex involved in telomere maintenance [43]. TINF2 displays two putative mitochondrial targeting sequences (MTS) in its N-terminus [44], one of which partially overlaps with a potential nuclear export signal (NES) motif according to the ELM resource [20]. By interacting with the N-terminal region of TINF2, ACD hides the three signals located in the same region that promote the export of TINF2 from the nucleus and its targeting to the mitochondrion, respectively [44]. Therefore, the ACD protein is responsible of the nuclear localisation of TINF2 [43, 44], and inhibits its mitochondrial localisation. Moreover, the TINF2 subcellular distribution may be determined by the ACD abundance. Importantly, functional assays on the mitochondrial pool of TINF2 showed that this protein moonlights as a regulator of oxidative phosphorylation and mitochondrial morphology [44]. Altogether, the presence of SLiMs is instrumental in the change of localisation and the subsequent change in function of the TINF2 protein in particular, and of the described moonlighting proteins in general.

\section{Databases of moonlighting and extreme multifunctional proteins}

Over the years, hundreds of moonlighting proteins have been identified experimentally. As of December 2018, there are three public databases containing multi-species collections of moonlighting and extreme multifunctional proteins described in the literature: MoonProt, MultitaskProtDB and MoonDB (Table 2). The MoonProt database [45] contains more than 350 proteins that pertain to the strict definition of moonlighting proteins (as described above) and were identified through manual curation of published articles providing experimental evidence supporting the presence of multiple functions. The MultitaskProtDB [7] stores more 
than 650 manually curated multifunctional proteins, not necessarily sticking to the strict definition of moonlighting proteins. Both databases provide the description of both canonical and additional functions including the PubMed identifier(s) of supporting experimental evidence, several protein annotations (e.g., Gene Ontology) and cross-references to other relevant databases such as OMIM [46] and the PDB [47]. Finally, MoonDB [48] contains 351 proteins that are both predicted EMF proteins as well as experimentally identified EMF or moonlighting proteins. Importantly, for each EMF protein, MoonDB specifies the pairs of dissimilar (i.e., unrelated) functions supporting the predictions. In addition, since the ability to perform unrelated functions can be due to the presence of a protein in unrelated subcellular locations [8], MoonDB lists the pairs of unrelated cellular components associated to each protein, identified by the PrOnto tool [18]. Finally, as for the other two resources, MoonDB provides functional annotations and many cross-references to other databases to fully describe moonlighting and EMF proteins.

\section{Conclusions}

We have described here several cases in which moonlighting is achieved through SLiMmediated regulatory switches. Recent computational analysis suggests that such type of regulation can be a prevalent strategy to increase cellular complexity by allowing single polypeptides to perform several unrelated functions. The elucidation of these regulatory mechanisms will enable a better comprehension of the crosstalk between cellular processes and cellular homeostasis. This is particularly important in the context of disease and disease comorbidities in which moonlighting and extreme multifunctional proteins play a central role $[5,9,49]$. Importantly, these regulatory mechanisms could offer an intervention strategy that can be used for the development of drugs targeting SLiMs. Indeed, small-molecule inhibitors 
and peptide mimetics have already shown promise in the manipulation of motif interactions [50].

\section{Notes}

\section{Acknowledgements}

The project leading to this publication has received funding from Excellence Initiative of AixMarseille University - A*MIDEX, a French "Investissements d'Avenir" programme (to CB).

\section{Tables}

Table 1. Representative examples of short linear motifs that have an effect on the localisation and function of known moonlighting proteins. MTS, mitochondrial targeting sequence; NES, nuclear export signal.

\begin{tabular}{|l|l|l|l|l|}
\hline $\begin{array}{l}\text { Moonlighting } \\
\text { protein }\end{array}$ & Type of short linear motif & Residue(s) & $\begin{array}{l}\text { Effect on localisation and/or } \\
\text { function }\end{array}$ & Reference(s) \\
\hline GAPDH & Nitrosylation site & C150 & Translocation to the nucleus & {$[29]$} \\
\hline GAPDH & Acetylation site & K160 & Cell death induction & {$[30]$} \\
\hline ACO1 & Phosphorylation site & S138 & Favour of the RNA-binding form & {$[33]$} \\
\hline ACO1 & Phosphorylation site & S138 & Destabilisation of the Fe-S cluster & {$[34]$} \\
\hline PGK1 & Phosphorylation site & Y76 & Decreased RNA-binding affinity & {$[36]$} \\
\hline DNM2 & Phosphorylation site & S764 & Cytokinesis regulation & {$[38,41]$} \\
\hline TINF2 & Targeting motif & $\begin{array}{l}1-58(M T S) \\
55-68 \\
(N E S)\end{array}$ & $\begin{array}{l}\text { Motif hiding and nuclear retention } \\
\text { through interaction with ACD }\end{array}$ & {$[44]$} \\
\hline
\end{tabular}


Table 2. Publicly available databases of moonlighting and multifunctional proteins.

\begin{tabular}{|l|l|l|l|l|l|}
\hline Database name & $\begin{array}{l}\text { Annotation } \\
\text { type }\end{array}$ & $\begin{array}{l}\text { Total number of } \\
\text { proteins }\end{array}$ & $\begin{array}{l}\text { Most } \\
\text { represented } \\
\text { organisms }\end{array}$ & Database URL & Reference \\
\hline MultitaskProtDB-II & $\begin{array}{l}\text { Manually } \\
\text { curated }\end{array}$ & 694 & $\begin{array}{l}\text { Human, } \\
\text { Yeast, } \\
\text { Arabidopsis }\end{array}$ & http://wallace.uab.es/multitaskII & {$[7]$} \\
\hline MoonProt & $\begin{array}{l}\text { Manually } \\
\text { curated }\end{array}$ & 361 & $\begin{array}{l}\text { Human, } \\
\text { Yeast, } \\
\text { several } \\
\text { bacterial } \\
\text { strains }\end{array}$ & http://moonlightingproteins.org/ & {$[45]$} \\
\hline MoonDB & $\begin{array}{l}\text { Manually } \\
\text { curated, } \\
\text { predicted }\end{array}$ & 351 & $\begin{array}{l}\text { Human, } \\
\text { Yeast, } \\
\text { Mouse }\end{array}$ & http://moondb.hb.univ-amu.fr/ & {$[48]$} \\
\hline
\end{tabular}

\section{References}

1. Beadle GW, Tatum EL (1941) Genetic Control of Biochemical Reactions in Neurospora. Proc Natl Acad Sci USA 27:499-506

2. Piatigorsky J, Wistow GJ (1989) Enzyme/crystallins: gene sharing as an evolutionary strategy. Cell 57:197-199

3. Jeffery CJ (1999) Moonlighting proteins. Trends Biochem Sci 24:8-11

4. Huberts DHEW, van der Klei IJ (2010) Moonlighting proteins: an intriguing mode of multitasking. Biochim Biophys Acta 1803:520-525. https://doi.org/10.1016/j.bbamcr.2010.01.022

5. Chapple CE, Robisson B, Spinelli L, et al (2015) Extreme multifunctional proteins identified from a human protein interaction network. Nat Commun 6:7412. https://doi.org/10.1038/ncomms8412

6. Chapple CE, Brun C (2015) Redefining protein moonlighting. Oncotarget 6:1681216813. https://doi.org/10.18632/oncotarget.4793

7. Franco-Serrano L, Hernández S, Calvo A, et al (2018) MultitaskProtDB-II: an update of a database of multitasking/moonlighting proteins. Nucleic Acids Res 46:D645-D648. https://doi.org/10.1093/nar/gkx1066

8. Copley SD (2012) Moonlighting is mainstream: paradigm adjustment required. BioEssays : news and reviews in molecular, cellular and developmental biology 34:57888. https://doi.org/10.1002/bies.201100191

9. Jeffery CJ (2018) Protein moonlighting: what is it, and why is it important? Philos Trans R Soc Lond, B, Biol Sci 373:. https://doi.org/10.1098/rstb.2016.0523 
10. Jeffery CJ (2014) An introduction to protein moonlighting. Biochem Soc Trans 42:16791683. https://doi.org/10.1042/BST20140226

11. Gancedo C, Flores C-L, Gancedo JM (2016) The Expanding Landscape of Moonlighting Proteins in Yeasts. Microbiol Mol Biol Rev 80:765-777. https://doi.org/10.1128/MMBR.00012-16

12. Espinosa-Cantú A, Ascencio D, Herrera-Basurto S, et al (2018) Protein Moonlighting Revealed by Noncatalytic Phenotypes of Yeast Enzymes. Genetics 208:419-431. https://doi.org/10.1534/genetics.117.300377

13. Khan I, Chitale M, Rayon C, Kihara D (2012) Evaluation of function predictions by PFP, ESG, and PSI-BLAST for moonlighting proteins. BMC Proc 6 Suppl 7:S5. https://doi.org/10.1186/1753-6561-6-S7-S5

14. Khan IK, Bhuiyan M, Kihara D (2017) DextMP: deep dive into text for predicting moonlighting proteins. Bioinformatics 33:i83-i91. https://doi.org/10.1093/bioinformatics/btx231

15. Khan IK, Kihara D (2016) Genome-scale prediction of moonlighting proteins using diverse protein association information. Bioinformatics 32:2281-2288. https://doi.org/10.1093/bioinformatics/btw166

16. Maxwell CA, McCarthy J, Turley E (2008) Cell-surface and mitotic-spindle RHAMM: moonlighting or dual oncogenic functions? J Cell Sci 121:925-932. https://doi.org/10.1242/jcs.022038

17. Becker E, Robisson B, Chapple CE, et al (2012) Multifunctional proteins revealed by overlapping clustering in protein interaction network. Bioinformatics 28:84-90. https://doi.org/10.1093/bioinformatics/btr621

18. Chapple CE, Herrmann C, Brun C (2015) PrOnto database: GO term functional dissimilarity inferred from biological data. Front Genet 6:200. https://doi.org/10.3389/fgene.2015.00200

19. Davey NE, Van Roey K, Weatheritt RJ, et al (2012) Attributes of short linear motifs. Molecular bioSystems 8:268-81. https://doi.org/10.1039/c1mb05231d

20. Gouw M, Michael S, Sámano-Sánchez H, et al (2018) The eukaryotic linear motif resource - 2018 update. Nucleic Acids Res 46:D428-D434. https://doi.org/10.1093/nar/gkx1077

21. Deribe YL, Pawson T, Dikic I (2010) Post-translational modifications in signal integration. Nature structural \& molecular biology 17:666-72. https://doi.org/10.1038/nsmb.1842

22. Perkins JR, Diboun I, Dessailly BH, et al (2010) Transient protein-protein interactions: structural, functional, and network properties. Structure 18:1233-1243. https://doi.org/10.1016/j.str.2010.08.007

23. Gibson TJ (2009) Cell regulation: determined to signal discrete cooperation. Trends Biochem Sci 34:471-482. https://doi.org/10.1016/j.tibs.2009.06.007

24. Neduva V, Russell RB (2005) Linear motifs: evolutionary interaction switches. FEBS letters 579:3342-5. https://doi.org/10.1016/j.febslet.2005.04.005 
25. Van Roey K, Gibson TJ, Davey NE (2012) Motif switches: decision-making in cell regulation. Curr Opin Struct Biol 22:378-385. https://doi.org/10.1016/j.sbi.2012.03.004

26. Brooks CL, Li M, Hu M, et al (2007) The p53--Mdm2--HAUSP complex is involved in p53 stabilization by HAUSP. Oncogene 26:7262-7266. https://doi.org/10.1038/sj.onc.1210531

27. Van Roey K, Dinkel H, Weatheritt RJ, et al (2013) The switches.ELM resource: a compendium of conditional regulatory interaction interfaces. Science signaling 6:rs7. https://doi.org/10.1126/scisignal.2003345

28. Tristan C, Shahani N, Sedlak TW, Sawa A (2011) The diverse functions of GAPDH: views from different subcellular compartments. Cell Signal 23:317-323. https://doi.org/10.1016/j.cellsig.2010.08.003

29. Hara MR, Agrawal N, Kim SF, et al (2005) S-nitrosylated GAPDH initiates apoptotic cell death by nuclear translocation following Siah1 binding. Nat Cell Biol 7:665-674. https://doi.org/10.1038/ncb1268

30. Sen N, Hara MR, Kornberg MD, et al (2008) Nitric oxide-induced nuclear GAPDH activates p300/CBP and mediates apoptosis. Nat Cell Biol 10:866-873. https://doi.org/10.1038/ncb1747

31. Volz K (2008) The functional duality of iron regulatory protein 1. Curr Opin Struct Biol 18:106-111. https://doi.org/10.1016/j.sbi.2007.12.010

32. Fillebeen C, Caltagirone A, Martelli A, et al (2005) IRP1 Ser-711 is a phosphorylation site, critical for regulation of RNA-binding and aconitase activities. Biochem J 388:143150. https://doi.org/10.1042/BJ20041623

33. Clarke SL, Vasanthakumar A, Anderson SA, et al (2006) Iron-responsive degradation of iron-regulatory protein 1 does not require the Fe-S cluster. EMBO J 25:544-553. https://doi.org/10.1038/sj.emboj.7600954

34. Deck KM, Vasanthakumar A, Anderson SA, et al (2009) Evidence that phosphorylation of iron regulatory protein 1 at Serine 138 destabilizes the [4Fe-4S] cluster in cytosolic aconitase by enhancing 4Fe-3Fe cycling. J Biol Chem 284:12701-12709. https://doi.org/10.1074/jbc.M807717200

35. Lay AJ, Jiang XM, Kisker O, et al (2000) Phosphoglycerate kinase acts in tumour angiogenesis as a disulphide reductase. Nature 408:869-873. https://doi.org/10.1038/35048596

36. Shetty P, Velusamy T, Bhandary YP, et al (2010) Urokinase receptor expression involves tyrosine phosphorylation of phosphoglycerate kinase. Mol Cell Biochem 335:235-247. https://doi.org/10.1007/s11010-009-0273-4

37. Royle SJ (2011) Mitotic moonlighting functions for membrane trafficking proteins. Traffic 12:791-798. https://doi.org/10.1111/j.1600-0854.2011.01184.x

38. Smith CM, Chircop M (2012) Clathrin-mediated endocytic proteins are involved in regulating mitotic progression and completion. Traffic 13:1628-1641. https://doi.org/10.1111/tra.12001 
39. Ferguson SM, De Camilli P (2012) Dynamin, a membrane-remodelling GTPase. Nat Rev Mol Cell Biol 13:75-88. https://doi.org/10.1038/nrm3266

40. Thompson HM, Skop AR, Euteneuer U, et al (2002) The large GTPase dynamin associates with the spindle midzone and is required for cytokinesis. Curr Biol 12:21112117

41. Chircop M, Sarcevic B, Larsen MR, et al (2011) Phosphorylation of dynamin II at serine764 is associated with cytokinesis. Biochim Biophys Acta 1813:1689-1699. https://doi.org/10.1016/j.bbamcr.2010.12.018

42. Monaghan RM, Whitmarsh AJ (2015) Mitochondrial Proteins Moonlighting in the Nucleus. Trends Biochem Sci 40:728-735. https://doi.org/10.1016/j.tibs.2015.10.003

43. Chen L-Y, Liu D, Songyang $Z$ (2007) Telomere maintenance through spatial control of telomeric proteins. Mol Cell Biol 27:5898-5909. https://doi.org/10.1128/MCB.00603-07

44. Chen L-Y, Zhang Y, Zhang Q, et al (2012) Mitochondrial localization of telomeric protein TIN2 links telomere regulation to metabolic control. Mol Cell 47:839-850. https://doi.org/10.1016/j.molcel.2012.07.002

45. Chen C, Zabad S, Liu H, et al (2018) MoonProt 2.0: an expansion and update of the moonlighting proteins database. Nucleic Acids Res 46:D640-D644. https://doi.org/10.1093/nar/gkx1043

46. Amberger JS, Bocchini CA, Schiettecatte F, et al (2015) OMIM.org: Online Mendelian Inheritance in Man (OMIM®), an online catalog of human genes and genetic disorders. Nucleic Acids Res 43:D789-798. https://doi.org/10.1093/nar/gku1205

47. wwPDB consortium (2018) Protein Data Bank: the single global archive for 3D macromolecular structure data. Nucleic Acids Res. https://doi.org/10.1093/nar/gky949

48. Ribeiro DM, Briere G, Bely B, et al (2018) MoonDB 2.0: an updated database of extreme multifunctional and moonlighting proteins. Nucleic Acids Res. https://doi.org/10.1093/nar/gky1039

49. Zanzoni A, Chapple CE, Brun C (2015) Relationships between predicted moonlighting proteins, human diseases, and comorbidities from a network perspective. Front Physiol 6:171. https://doi.org/10.3389/fphys.2015.00171

50. Corbi-Verge C, Kim PM (2016) Motif mediated protein-protein interactions as drug targets. Cell Commun Signal 14:8. https://doi.org/10.1186/s12964-016-0131-4 